\title{
Carnosine and Anserine in Chicken: Distribution, Age-dependency and their Anti-glycation Activity
}

\author{
Seung-Ki Kim, Yumi Kim, In Kee Baek', and Joong-Hyuck Auh* \\ Department of Food Science and Technology, Chung-Ang University, Anseong 465-756, Korea \\ ${ }^{1}$ Department of Animal Science and Technology, Chung-Ang University, Anseong 465-756, Korea
}

\begin{abstract}
The imidazole dipeptide carnosine and its methylated anserine analogues are the major histidine containing dipeptides in vertebrate tissue, especially in skeletal muscle, the heart, and the central nervous system. In this study, the carnosine and anserine content in chicken from different parts and of differing ages was determined and their physiological activities were compared. Anserine was more dominant than carnosine in these tissues and both of them significantly decreased with aging in all parts of chicken muscles. Chicken breast muscle showed the highest content of carnosine and anserine than drumstick and wing. Advanced glycated end-product (AGE) formation was inhibited up to $60 \%$ by the extract from 20 wk chicken breast and decreased with aging $(90 \mathrm{wk})$. Anti-oxidation activity was also significantly reduced from $61.2 \%$ to $52.9 \%$ with aging. As results, anti-glycation and anti-oxidation activity of carnosine and anserine extract from chicken muscle increased proportionally to the amount of those peptides in the muscle, while these decreased with the aging process.
\end{abstract}

Key words: carnosine, anserine, advanced glycated end-product, anti-glycation activity, chicken

\section{Introduction}

Carnosine is a natural dipeptide containing of $\beta$-alanine and L-histidine. The imidazole dipeptide carnosine and its methylated analogues anserine are major histidine containing dipeptides in vertebrate animal (chicken, pork, cattle and so on) tissue, especially in skeletal muscle, the heart, and the central nervous system (Mora et al., 2008; Tian et al., 2007). It has been known to play a role in quite a number of physiological functions in vertebrates. Carnosine's role includes those of physiological buffer in vertebrate's muscle to offset the production of lactic acid during exercise, inhibition of oxidative reaction in hydroxyl-radical and singlet oxygen-scavenging and lipid peroxidation (Intarapichet and Maikhunthod, 2005; Lee et al., 1998), inhibition of advanced glycated end-product (AGE) formation (Dukic-Stefanovic et al., 2001), neurotransmitter in brain (Hipkiss and Brownson, 2000), regulation of enzyme activity, and chelate prooxidative metals (Decker et al., 2000). Since the first isolation of

*Corresponding author: Joong-Hyuck Auh, Department of Food Science and Technology, Chung-Ang University, Anseong 456-756, Korea. Tel: 82-31-670-3079, Fax: 82-31-675-4853, E-mail: jhauh@cau.ac.k carnosine from beef muscle extract in 1900s, quantitative determination of carnosine from muscle food has been a focus of interest and the potential of carnosine as a natural food supplement for those with poor health has been getting an interest as well (Hipkiss et al., 2000; Mora et al., 2008). For example, carnosine and anserine have been known as good anti-oxidants, preventing of diseases resulting from lipid oxidation in skeletal muscle (Chan and Decker, 1994). Their physiological activities were exhibited through buffering capacity (Kohen et al., 1988), reducing chelating pro-oxidant metals (Decker et al., 2000), singlet oxygen (Dahl et al., 1988) and peroxy radical (Kohen et al., 1988).

Carnosine has been found with higher concentration in white muscle than red muscle tissue (Boldyrev et al., 2004; Brown., 1981; Hipkiss, 2005; Plowman and Close, 1988). Chicken is a representative animal with higher carnosine content among the white muscle tissue animals (Boldyrev and Severin, 1990). However, no systemic investigation was attempted on the changes of carnosine and anserine levels in chicken muscle with aging. In this study, we compared the content of carnosine and anserine from different parts and ages of chicken muscles along with their physiological (anti-glycation and anti-oxidation) activities. 


\section{Materials and Methods}

\section{Chemicals and reagents}

All the reagents used in this study were of the highest purity available. Acetonitrile (ACN), methanol, bovine serum albumin (BSA), methylglyoxal, L-ascorbic acid, $\alpha, \alpha$-diphenyl- $\beta$-picrylhydrazyl (DPPH), $35 \%$ hydrochloric acid, sodium chloride, boric acid, $o$-phthaldialdehyde (OPA), L-carnosine, and L-anserine were purchased from Sigma Aldrich Co. (USA).

\section{Extraction of carnosine and anserine from chicken muscle}

Chicken samples (20 and $90 \mathrm{wk}$ laying hen) were received from Department of Animal Science and Technology, Chung-Ang University, Korea. At the end of each week, chickens were sacrificed by cervical dislocation, a method approved by Animal Care Committee of ChungAng University. Carnosine and anserine was extracted according to Auh et al. (2010) with slight modification. Muscle samples from three different parts (Breast, Drumstick, and Wing) were taken and freeze-dried for $72 \mathrm{~h}$. Dried muscle samples were finely ground and $0.5 \mathrm{~g}$ of ground muscle was suspended in $12 \mathrm{~mL}$ of distilled water and incubated for $2 \mathrm{~h}$ at $25^{\circ} \mathrm{C}$ with moderate shaking. Subsequently, the mixture was centrifuged at $14000 \mathrm{~g}$ for $30 \mathrm{~min}$ and $300 \mu \mathrm{L}$ of supernatant was mixed with 900 $\mu \mathrm{L}$ of cold ethanol, then kept for $20 \mathrm{~min}$ at $4^{\circ} \mathrm{C}$. Supernatant taken after centrifugation at $14000 \mathrm{~g}$ for $30 \mathrm{~min}$ was filtered through membrane syringe filter (MCE type, 0.45 $\mathrm{mm}$, Adavantec, MFS Inc., Japan) and freeze dried. Lyophilized samples were kept at $-40^{\circ} \mathrm{C}$ until analysis.

\section{HPLC analysis}

Carnosine and anserine concentration was determined according to Aristoy et al. (2004) and Auh et al. (2010). The HPLC system (Gilson Medical Electronics, USA) equipped with a fluorescence detector (Agilent, USA), a column oven and a Spherisorb SCX column $(4.6 \times 250$ $\mathrm{mm}$, Waters Co., USA) was used for the analysis. Ten $\mu \mathrm{L}$ of the sample solution $(10 \%, \mathrm{w} / \mathrm{v})$ was mixed with 100 $\mu \mathrm{L}$ of OPA solution and placed for $2 \mathrm{~min}$ at room temperature under dark. Then, $20 \mu \mathrm{L}$ of derivatized samples was injected into HPLC. Mobile phase was composed of $80 \%$ of phase A $(20 \%$ acetonitrile in $6.6 \mathrm{mM}$ hydrochloric acid) and $20 \%$ of phase B (phase A containing $0.8 \mathrm{M}$ sodium chloride), eluted as $0.6 \mathrm{~mL} / \mathrm{min}$ for $30 \mathrm{~min}$ at $40^{\circ} \mathrm{C}$. For the fluorescence detection, 338 and $445 \mathrm{~nm}$ were used as excitation and emission wavelengths, respectively.

\section{Anti-glycation activity (AGE inhibition)}

Anti-glycation activity of carnosine containing extract from chicken breast (20 and 90 wk laying hen) was measured by $\%$ inhibition of advanced glycated end-product (AGE) formation according to Li et al. (2008) with modification. Extract was mixed with bovine serum albumin (BSA, $50 \mathrm{mg} / \mathrm{mL}$ ) and methylglyoxal $(3 \mathrm{mM})$ in $50 \mathrm{mM}$ sodium phosphate buffer ( $\mathrm{pH}$ 7.4) and incubated for $24 \mathrm{~h}$ in dark condition. The formation of AGE was quantified by measuring the fluorescence intensity (excitation at 350 $\mathrm{nm}$, emission $450 \mathrm{~nm}$ ) using Spectrofluorimeter RF-1500 (Shimadzu, Japan). All the measurement was conducted in triplicates.

\section{Anti-oxidation activity (DPPH radical scavenging effect)}

Anti-oxidation of carnosine containing extract from chicken breast (20 and $90 \mathrm{wk}$ laying hen) extracts on $\alpha, \alpha$ diphenyl- $\beta$-picrylhydrazyl (DPPH) radicals was measured with a method described by Wu et al. (2003) with modification. Samples were mixed with $0.2 \mathrm{mM}$ DPPH (in $95 \%$ methanol), kept for $30 \mathrm{~min}$ at room temperature and the absorbance at $517 \mathrm{~nm}$ was measured and anti-oxidation activity was calculated. All the measurements were conducted in triplicates.

\section{Statistical analysis}

All the experimental data were analyzed using the ANOVA procedure of SAS (SAS Institute Inc., USA) and the significance was defined at $p<0.05$.

\section{Results and Discussion}

\section{Determination of carnosine and anserine in chicken}

Carnosine and anserine in chicken were determined by HPLC after OPA pre-column derivatization. The carnosine and anserine content of chicken from different parts and ages were summarized in Table 1. In general, anserine was more dominant than carnosine and both of them significantly decreased with aging in all parts of chicken muscles. Chicken breast parts showed the highest content of carnosine and anserine followed by drumstick and wing. It was in good agreement with previous report by Intarapichet and Maikhunthod (2005) stated that chicken breast muscle had more carnosine than other parts of chicken. Carnosine and anserine in drumstick muscle was seriously affected by aging, but no significant decrease 
Table 1. Concentration of carnosine and anserine in chickens from different parts and ages

\begin{tabular}{|c|c|c|c|}
\hline & Parts & $\begin{array}{l}\text { Carnosine concen- } \\
\text { tration }(\mathrm{mg} / \mathrm{kg})\end{array}$ & $\begin{array}{c}\text { Anserine concen- } \\
\text { tration }(\mathrm{mg} / \mathrm{kg})\end{array}$ \\
\hline \multirow{3}{*}{$\begin{array}{c}20 \mathrm{wk} \\
\text { laying hen }\end{array}$} & Breast & $712.64 \pm 22.38^{\mathrm{a}}$ & $3367.71 \pm 90.62^{\mathrm{A}}$ \\
\hline & Drumstick & $395.97 \pm 17.40^{c}$ & $1165.23 \pm 49.51^{\mathrm{E}}$ \\
\hline & Wing & $384.19 \pm 32.52^{\mathrm{c}}$ & $2594.99 \pm 92.41^{\mathrm{C}}$ \\
\hline \multirow{3}{*}{$\begin{array}{c}90 \mathrm{wk} \\
\text { laying hen }\end{array}$} & Breast & $553.55 \pm 13.81^{b}$ & $2915.54 \pm 12.01^{\mathrm{B}}$ \\
\hline & Drumstick & $142.79 \pm 11.23^{\mathrm{d}}$ & $693.04 \pm 18.91^{\mathrm{F}}$ \\
\hline & Wing & $359.17 \pm 11.37^{\mathrm{c}}$ & $2264.86 \pm 15.84^{\mathrm{D}}$ \\
\hline
\end{tabular}

was observed in the wing muscle. It implied that the decrease of carnosine was proportional to the ratio of white to red muscle in the samples. More decrease of carnosine content was observed in white muscle (breast) than red muscle (wing).

\section{Anti-glycation and anti-oxidation activity (AGE inhibition)}

Anti-glycation activity of chicken breast extract was measured by \% inhibition of advanced glycated endproduct (AGE) formation. AGE formation is increasing with aging as well as in diabetes mellitus, which play an important role in the development of chronic diabetic complications (Ahmed, 2005). Although numerous agents for AGE inhibition have been developed, side effects and cost are limiting the application of the inhibiting agents in practice. The chicken breast extract containing carnosine and anserine showed nice AGE inhibition and the results are summarized in Fig. 1. AGE formation was inhibited up to $60 \%$ with the extract from 20 wk chicken breast and decreased with aging $(90 \mathrm{wk}$ ), which meant the more accumulation of AGE in muscle as aging proceed. Antioxidation activity of carnosine containing peptide mixture derived from chicken breast was measured by DPPH radical scavenging effect (Fig. 1). The activity significantly decreased from $61.2 \%$ to $52.9 \%$ with aging, which might be influenced by the reduction of carnosine and anserine content in the muscle. Although anserine and carnosine are interrelated in physiologically, more research has been done on carnosine to date. But, Wu et al. (2003) indicated that anserine had similar radical scavenging effect and higher reducing power compared to carnosine. Therefore, anserine content should be considered along with carnosine for the anti-oxidation activity. As a result, anti-glycation and anti-oxidation activity of carnosine and anserine extract from chicken muscle increase proportionally to

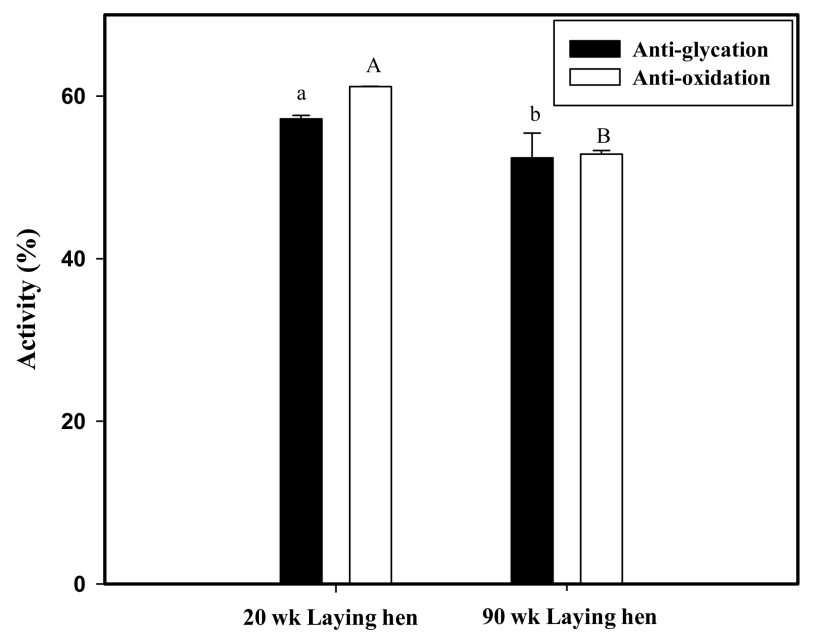

Fig. 1. Anti-glycation and anti-oxidation activities of the carnosine and anserine extract from chicken breast from different ages.

the amount of those peptides in the muscle.

In conclusion, we revealed that the carnosine and anserine content in chicken decreased with aging and the degree of reduction increased in proportion to the ratio of white muscles in each tissue. Although the chicken breast muscle was reported as a good source of carnosine (Intarapichet and Maikhunthod, 2005), anserine was more predominant than carnosine as indicated by Auh et al. (2010) and Huang and Kuo (2000). Anti-glycating and anti-oxidation, typical physiological activities of carnosine and anserine, significantly decreased by aging, however, they exhibited sufficient levels of activities even in 90 wk old. This is the first report regarding the distribution of carnosine and anserine in chicken and the effect of aging on it. This study would be a solid basement for the development of specialized functional meat product by the physiological functions of carnosine and anserine.

\section{Acknowledgments}

This study was supported by Chung-Ang University excellent freshman scholarship grants and Technology Development Program for Agriculture and Forestry, Ministry for Food, Agriculture, Forestry and Fisheries, Republic of Korea.

\section{References}

1. Ahmed, N. (2005) Advanced glycation end products-role in pathology of diabetic complications. Diabetes Res. Clin. Pr. 67, 3-21.

2. Aristoy, M. C. and Toldrá, F. (2004) Histidine dipeptides HPLC- 
based test for the detection of mammalian origin proteins in feeds for ruminants. Meat Sci. 67, 211-217.

3. Auh, J. H., Namgung, N., Shin, K. S., Park, S. W., and Paik, I. K. (2010) Effects of supplementary blood meal on the content of carnosine and anserine in broiler meat. J. Poult. Sci. 47, 302-309.

4. Boldyrev, A. A. and Severin, S. E. (1990) The histidine containing dipeptides, carnosine and anserine: Distribution, properties and biological significance. In: Advences in Enzyme Regulation. Weber, G (ed) Pergamon Press, London, Vol. 30, pp. 175-194.

5. Boldyrev, A. A., Bulygina, E., Leinsoo, T., Petrushanko, I., Tsubone, S., and Abe, H. (2004) Protection of neuronal cells against reactive weaned oxygen species by carnosine and related compounds. Comp. Biochem. Physiol. B: Biochem. Mol. Biol. 137, 81-88.

6. Brown, C. E. (1981) Interactions among carnosine, anserine, ophidine and copper in biochemical adaptation. J. Theor. Biol. 88, 245-256.

7. Chan, K. M. and Decker, E. A. (1994) Endogenous skeletal muscle antioxidants. Crit. Rev. Food Sci. Nutr. 34, 403-426.

8. Dahl, T. A., Midden, W. R., and Hartman, P. E. (1988) Some prevalents biomolecules as defenses against singlet oxygen damage. Photochem. Photobiol. 47, 357-362.

9. Decker, E. A., Livisay, S. A., and Zhou, S. (2000) A re-evaluation of the antioxidant activity of purified carnosine. Biochem. 7, 901-906.

10. Dukic-Stefanovic, S., Schinzel, R., Riederer, P., and Münch, G. (2001) AGEs in brain ageing: AGE-inhibitors as neuroprotective and anti-dementia drugs?. Biogerontol. 2, 19-34.

11. Hipkiss, A. R. and Brownson, C. (2000) A possible new role for the anti-ageing peptide carnosine. Cell. Mol. Life Sci. 57, 747-753.

12. Huang, S. C. and Kuo, J. C. C. (2000) Concentration and antioxidative activity of anserine and carnosine in poultry meat extracts treated with demineralization and papain. Proc. Natl. Sci. Counc. Republic China B Life Sci. 24, 193201.

13. Intarapichet, K. O. and Maikhunthod, B. (2005) Genotype and gender differences in carnosine extracts and antioxidant activities of chicken breast and thigh meats. Meat Sci. 71, 634-642.

14. Kohen, R., Yamamoto, Y., Cundy, K. C., and Ames, B. N. (1988) Antioxidant activity of carnosine, homocarnosine, and anserine present in muscle and brain. Proc. Natl. Acad. Sci. USA. 85, 3175-3179.

15. Lee, C. J., Yim, M. B., Chock, P. B., Yim, H. S., and Kang, S. O. (1998) Oxidation-reduction properties of methylglyoxal-modified protein in relation to free radical generation. $J$. Biol. Chem. 273, 25272-25278.

16. Li, W., Ota, K., Nakamura, J., Naruse, K., Nakashima, E., Oiso, Y., and Hamada, Y. (2008) Antiglycation effect of gliclazide on in vitro AGE formation from glucose and methylglyoxal. Exp. Biol. Med. 233, 176-179.

17. Mora, L., Sentandreu, M. A., and Toldrá, F. (2008) Contents of creatine, creatinine and carnosine in porcine muscles of different metabolic types. Meat Sci. 79, 709-715.

18. Plowman, J. E. and Close, E. A. (1988) An evaluation of a method to dierentiate the species of origin of meats on the basis of the contents of anserine, balenine and carnosine in skeletal muscle. J. Agric. Food Chem.45, 69-78.

19. Tian, Y., Xie, M., Wang, W., Wu, H., Fu, Z., and Lin, L. (2007) Determination of carnosine in black-bone silky fowl (Gallus gallus domesticus Brisson) and common chicken by HPLC. Eur. Food Res. Technol. 226, 311-314.

20. Wu, H. C., Shiau, C. Y., Chen, H. M., and Chiou, T. K. (2003) Antioxidant activities of carnosine, anserine, some free amino acids and their combination. J. Food Drug Anal. 11, 148-153.

(Resived 2011.12.30/Revised 2012.1.10/Accepted 2012.1.18) 